\title{
A CFD-Aided Galerkin Method for Global Linear Instability Analysis
}

\author{
Shengqi Zhang ${ }^{1}$, Zhenhua $\mathrm{Xia}^{2, *}$ and Shiyi Chen ${ }^{3,1}$ \\ 1 State Key Laboratory for Turbulence and Complex System, Peking University, \\ Beijing, China. \\ ${ }^{2}$ Department of Engineering Mechanics, Zhejiang University, Zhejiang, China. \\ ${ }^{3}$ Department of Mechanics and Aerospace Engineering, Southern University of \\ Science and Technology, Shenzhen, China.
}

Received 27 February 2020; Accepted (in revised version) 12 May 2020

\begin{abstract}
Global linear instability analysis is a powerful tool for the complex flow diagnosis. However, the methods used in the past would generally suffer from some disadvantages, either the excessive computational resources for the low-order methods or the tedious mathematical derivations for the high-order methods. The present work proposed a CFD-aided Galerkin methodology which combines the merits from both the low-order and high-order methods, where the expansion on proper basis functions is preserved to ensure a small matrix size, while the differentials, incompressibility constraints and boundary conditions are realized by applying the low-order linearized Navier-Stokes equation solvers on the basis functions on a fine grid. Several test cases have shown that the new method can get satisfactory results for onedimensional, two-dimensional and three-dimensional flow problems and also for the problems with complex geometries and boundary conditions.
\end{abstract}

AMS subject classifications: 76Exx

Key words: Global linear instability, spatial discretization, Galerkin method.

\section{Introduction}

Flow instability analysis plays a key role in understanding the origin of many complex fluid motions including turbulence. Among all kinds of instability analysis methods,

\footnotetext{
*Corresponding author. Email addresses: 1801111635@pku.edu.cn (S. Zhang), xiazh@zju.edu.cn (Z. Xia), chensy@sustech.edu.cn (S. Chen) 
linear instability analysis is the simplest and most widely used one, and it can obtain satisfactory results in shear flows, rotating flows, buoyancy-driven convections and surfacetension-driven instability with steady and laminar basic state. The classic Tollmien's theory and Orr-Sommerfeld equation can deal with the linear instability of one-dimensional parallel flow [1-4]. Consideration of another slowly evolving spatial direction will result in parabolised stability equations [5-7]. However, when the base flow is strongly inhomogeneous in more than one direction, the general global instability should be taken into account and the governing equations are no longer one-dimensional, raising the challenges on the establishment and the solution of respective eigenvalue problems.

Pierrehumbert and Widnall (1982) [8] published the pioneering work on global linear instability and discussed the instability of shear-layer vortices using spectral collocation method. Since then, the spectral collocation method and the spectral Galerkin method have been widely used $[9,10]$ for simple flow problems due to the small matrix size which benefits from the high-order accuracy of the methods. For piecewise regular domains such as backward facing step, multi-domain technique can be applied [11]. For more general geometry, spectral element method [12] and spectral/hp method [13] can be introduced as the generalization of multi-domain spectral method. Many other investigations using spectral method can be seen in [7,14-22]. Although global spectral methods are accurate and more likely to provide a small matrix for the full eigen-spectrum computation, the mathematical derivation is often tedious, as can be seen in [17].

Due to the simplicity and flexibility, simple discretization methods, including the finite difference method [23-28], the finite element method [29-31], the finite volume method $[25,32,33]$ and the lattice Boltzmann method $[34,35]$, have also been used to study the global linear instability. In order to make the analysis accurate enough, the simple low-order discretization methods would require large matrix size to fully characterize the linear operator. With high-order finite difference schemes, the required matrix size can be reduced [36], but it is still larger than those obtained in high-order spectral methods.

Although matrix-free subspace iteration approach [37] can be applied to obtain the leading eigen-modes, the computation expenses will increase heavily if more highaccuracy modes are required. Therefore, matrix-forming approach is still the most reasonable choice on condition that the matrix size is as small as possible. As suggested by Theofilis [38], full eigenspectrum should be derived if possible because it is helpful for a deep understanding of the problem. In fact, there are still some interesting problems with simple geometry which can be analyzed with spectral methods (smallest matrix size required), but it is hard to realize due to the extremely tedious derivations.

The present work is devoted to finding a new way to construct the eigenproblem matrix which represents the linear operator. In the newly proposed method, the matrix is formed with the help of globally smooth basis functions while the differential operations are realized with the aid of the linearized CFD code. The remainder of the paper is organized as follows. Section 2 introduces the general problem setup and numerical method in detail. The new method will be validated in Section 3 where several one-dimensional 
(1D), two-dimensional (2D) and three-dimensional (3D) test cases are used to show its accuracy. Section 4 summarizes the present work.

\section{Numerical method}

\subsection{Basic concepts}

Considering the three-dimensional incompressible Navier-Stokes (NS) equation as the basic governing equations (the constant density is absorbed in pressure)

$$
\begin{aligned}
& \frac{\partial \boldsymbol{u}}{\partial t}+\boldsymbol{u} \cdot \nabla \boldsymbol{u}=-\nabla p+v \Delta \boldsymbol{u}, \\
& \nabla \cdot \boldsymbol{u}=0, \\
& \mathcal{D}_{\Sigma}\left(\boldsymbol{u} ; \boldsymbol{x}_{\Sigma}\right)=f_{\Sigma}\left(\boldsymbol{x}_{\Sigma}\right) .
\end{aligned}
$$

Here $v$ is the kinematic viscosity and $\mathcal{D}_{\Sigma}\left(\boldsymbol{u} ; \boldsymbol{x}_{\Sigma}\right)$ is the linear spatial differential operator defined at the boundary $\Sigma$.

With basis flow $\boldsymbol{u}_{0}$, the governing equation of the infinitesimal perturbation $\boldsymbol{u}^{\prime}$ can be described by the linearized Navier-Stokes (LNS) equation

$$
\begin{aligned}
& \frac{\partial \boldsymbol{u}^{\prime}}{\partial t}+\boldsymbol{u}_{0} \cdot \nabla \boldsymbol{u}^{\prime}+\boldsymbol{u}^{\prime} \cdot \nabla \boldsymbol{u}_{0}-\nabla p^{\prime}+v \Delta \boldsymbol{u}^{\prime} \\
& \nabla \cdot \boldsymbol{u}^{\prime}=0 \\
& \mathcal{D}_{\Sigma}\left(\boldsymbol{u}^{\prime} ; \boldsymbol{x}_{\Sigma}\right)=0 .
\end{aligned}
$$

After writing the variables into a one-column vector, the LNS equation can be rewritten as

$$
\begin{aligned}
\frac{\partial U}{\partial t} & =\frac{\partial}{\partial t}\left[\begin{array}{c}
u^{\prime} \\
v^{\prime} \\
w^{\prime}
\end{array}\right] \\
& =\mathcal{P}_{\Sigma} \mathcal{P}_{\perp}\left\{-\left[\begin{array}{lll}
\partial_{x} u_{0} & \partial_{y} u_{0} & \partial_{z} u_{0} \\
\partial_{x} v_{0} & \partial_{y} v_{0} & \partial_{z} v_{0} \\
\partial_{x} w_{0} & \partial_{y} w_{0} & \partial_{z} w_{0}
\end{array}\right]-\boldsymbol{u}_{0} \cdot \nabla+v \Delta\right\} \mathcal{P}_{\Sigma}\left[\begin{array}{c}
u^{\prime} \\
v^{\prime} \\
w^{\prime}
\end{array}\right] \\
& \triangleq \mathcal{P}_{\Sigma} \mathcal{D} \mathcal{P}_{\Sigma}\left[\begin{array}{c}
u^{\prime} \\
v^{\prime} \\
w^{\prime}
\end{array}\right] \\
& \triangleq \mathcal{L}(U)
\end{aligned}
$$

where $\mathcal{P}_{\Sigma}$ is the boundary condition operator setting specific linear differentials at boundary to zero, and $\mathcal{P}_{\perp}$ the projection operator making the field satisfy the incompressible conditions. Physically and mathematically, the initial discontinuity caused by boundary condition operator would be smoothed immediately by the viscosity. 
In general, a smooth flow field can be expanded with a series of smooth basis functions $\phi_{i}, i=1,2, \cdots$ with the specified inner product $\langle\cdot, \cdot\rangle$. The basis functions should be chosen properly (but they are not required to satisfy boundary conditions or other physical constraints) so that only a few terms could be enough to approximate the flow field

$$
U(\boldsymbol{x})=\sum_{i=1}^{\infty} \alpha_{i} \phi_{i}(\boldsymbol{x}) \approx \sum_{i=1}^{n} \alpha_{i} \phi_{i}(\boldsymbol{x}) .
$$

The linear operator $\mathcal{L}$ acting on one basis function could be described with a matrix in the space of $n$ basis functions

$$
\mathcal{L}\left(\phi_{i}\right) \approx \sum_{i=1}^{n} L_{j i} \phi_{j}
$$

with

$$
\begin{aligned}
& L=S^{-1} L^{*}, \\
& S_{i j} \triangleq\left\langle\phi_{i}, \phi_{j}\right\rangle, \\
& L_{j i}^{*} \triangleq\left\langle\mathcal{L}\left(\phi_{i}\right), \phi_{j}\right\rangle .
\end{aligned}
$$

If the basis functions are orthogonal with $S_{i j}=\delta_{i j}$, $\left(\delta_{i j}\right.$ is the Kronecker tensor), then $L=L^{*}$. With the above expansion, the linear operator acting on a field could be described with a matrix-vector product for the coefficients

$$
\begin{aligned}
\mathcal{L}(U) & \approx \mathcal{L}\left(\sum_{j=1}^{n} \alpha_{j} \phi_{j}\right)=\sum_{j=1}^{n} \alpha_{j} \mathcal{L}\left(\phi_{j}\right) \\
& \approx \sum_{j=1}^{n} \alpha_{j} \sum_{i=1}^{n} L_{i j} \phi_{i}=\sum_{i=1}^{n} \sum_{j=1}^{n} L_{i j} \alpha_{j} \phi_{i} .
\end{aligned}
$$

Therefore the expansion coefficient vector $\hat{\boldsymbol{\alpha}}=\left[\hat{\alpha}_{1}, \hat{\alpha}_{2}, \cdots, \hat{\alpha}_{n}\right]^{T}$ of an eigenfunction of $\mathcal{L}$ should satisfy the eigenvalue problem

$$
L \hat{\boldsymbol{\alpha}}=\lambda \hat{\boldsymbol{\alpha}},
$$

or the equivalent generalized eigenvalue problem

$$
L^{*} \hat{\boldsymbol{\alpha}}=\lambda S \hat{\boldsymbol{\alpha}}
$$

Considering the relationship (2.6), with a black box $\widetilde{\mathcal{L}}=\widetilde{\mathcal{P}}_{\Sigma} \widetilde{\mathcal{D}} \widetilde{\mathcal{P}}_{\Sigma}$ approximating operator $\mathcal{L}$, the $j$-th column of the matrix $L^{*}$ can be derived by doing inner product $\left\langle\phi_{i}, \widetilde{\mathcal{L}}\left(\phi_{j}\right)\right\rangle$ with $i$ from 1 to $n$.

For a traditional spectral Galerkin method, the black box is the exact $\mathcal{L}$ and the matrix is constructed with the above procedure. However, it demands the basis functions to satisfy boundary conditions in order to avoid the discontinuity or singularity. For low-order 
methods, the basis functions are chosen to be piecewise polynomials which are consistent with the numerical scheme. Considering a $N_{x} \times N_{y} \times N_{z}$ grid, the coefficients form a vector with $N_{x} \times N_{y} \times N_{z}$ entries. Each column of the matrix $L^{*}$ could be derived from a grid field resulting from the time derivative computed with LNS code. After $N_{b} \times N_{x} \times N_{y} \times N_{z}$ times of execution of the LNS code, an $\left(N_{b} \times N_{x} \times N_{y} \times N_{z}\right) \times\left(N_{b} \times N_{x} \times N_{y} \times N_{z}\right)\left(N_{b}\right.$ being the number of variables) matrix could be formed. Even for a small grid number $N_{x}=N_{y}=N_{z}=100$ and three variables $N_{b}=3$, the low-order method would create a matrix with $9 \times 10^{12}$ entries, which is very inconvenient even for the iterative eigensolvers.

In the present work, we proposed a new method which combines the merits from both the Galerkin spectral approach and the low-order methods. In the new method, the expansion on the basis functions is preserved to ensure a small matrix size, while the differentials, incompressibility constraints and boundary conditions are realized by applying the low-order LNS solvers on the basis functions on a fine grid without increasing the degree-of-freedom or the size of the derived matrix. However, if the structures in perturbation modes become increasingly tight with increasing Reynolds number while the number of the basis functions is fixed, the expansion residual would increase drastically because of the high algebraic precision. Therefore the resolution (i.e. the number of the basis functions) of the spectral expansion must be abundant for the present method, as what is required for a traditional spectral method.

\subsection{Basic numerical procedure}

Choosing one simple and grid-based discretization method (finite difference, finite volume, finite element or lattice Boltzmann) to discretize the LNS with imposing boundary conditions as the black box, the basic procedure of the newly proposed method to compute the matrix $L_{i j}^{*}$ and $S_{i j}$ is straightforward as sketched in Fig. 1 and can be written in pseudo code as follows:

FOR $i=1,2, \cdots, n$

i Get basis function $\phi_{i}(x)$ explicitly;

ii Specify grid values $\phi_{i}\left(x_{k}, y_{l}, z_{m}\right)$ at point $\left(x_{k}, y_{l}, z_{m}\right)$;

iii Apply LNS code to derive $\left[\widetilde{\mathcal{L}}\left(\phi_{i}\right)\right]\left(x_{k}, y_{l}, z_{m}\right)$;

iv Get interpolant $\left[\widetilde{\mathcal{L}}\left(\phi_{i}\right)\right](\boldsymbol{x})$;

v FOR $j=1,2, \cdots, n$

Compute $L_{j i}^{*}=\left\langle\widetilde{\mathcal{L}}\left(\phi_{i}\right), \phi_{j}\right\rangle$ and $S_{i j}=\left\langle\phi_{i}, \phi_{j}\right\rangle$.

END

END 


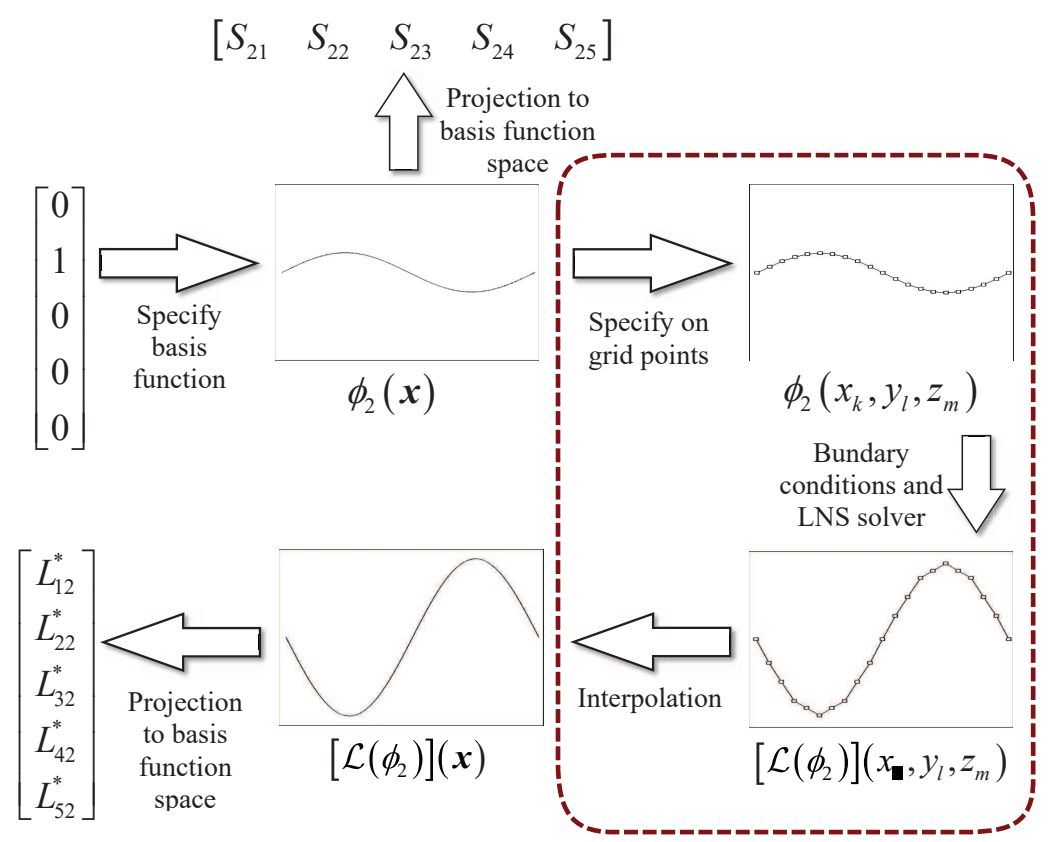

Figure 1: Procedure of computing a column of matrix $L^{*}$ and a row of matrix $S$ using the new method.

It should be noted that the step (iv) is not necessary to be done explicitly, since one can also compute $L_{j i}^{*}$ and $S_{i j}$ by doing numerical integrations directly on the discretization points. If the basis functions are of some special kinds, such as the Chebyshev polynomials, the step (iv) on the special collocation points could be used to promote the accuracy and the speed of the calculation.

\section{Numerical results}

\subsection{D heat equation}

Let's first consider a 1D linear heat transfer equation with Dirichlet boundary condition on both sides

$$
\begin{aligned}
& \frac{\partial \theta}{\partial t}=\frac{\partial^{2} \theta}{\partial x^{2}}, \quad x \in[0,1], \\
& \theta(0, t)=\theta(1, t)=0 .
\end{aligned}
$$

In this problem, the theoretical eigenvalues and eigenmodes are

$$
\left\{\begin{array}{l}
\sigma_{0}^{(k)}=-k^{2} \pi^{2}, \\
\theta_{0}^{(k)}=\sin (k \pi x), \quad k=1,2, \cdots .
\end{array}\right.
$$



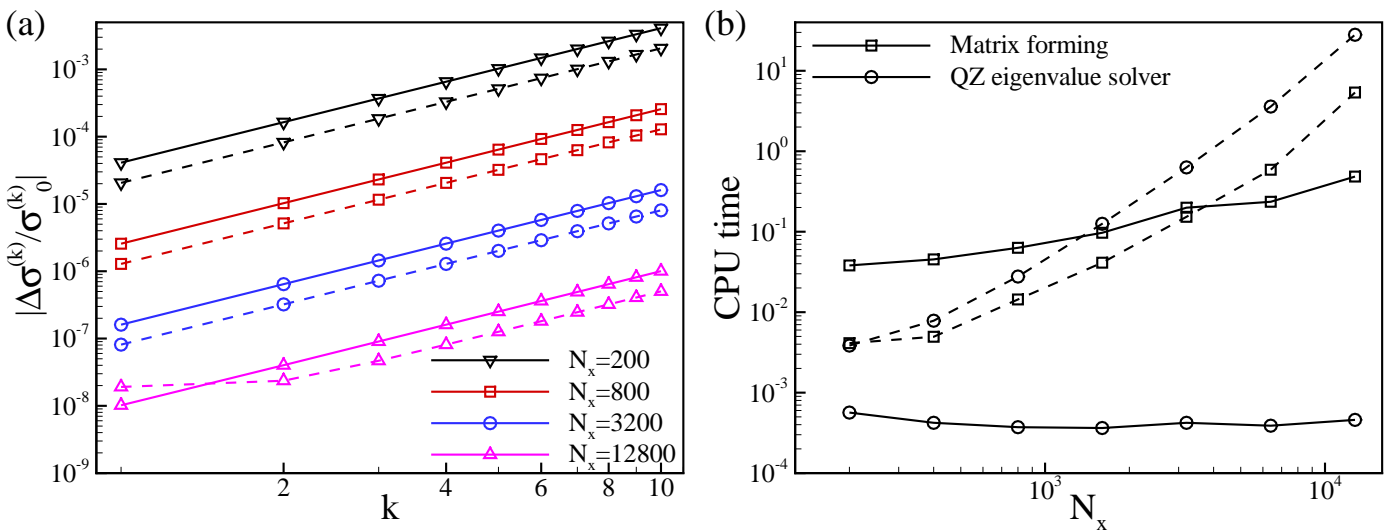

Figure 2: Comparison of the accuracy and efficiency of the new method and the traditional finite difference method. Solid lines refer to the new method and dashed lines refer to finite difference method. (a) Relative errors between computed and theoretical eigenvalues. (b) Execution time spent in the matrix formation and eigenvalue computation processes. Computation is performed with MATLAB R2018b on Intel(R) Core(TM) i7-2600 3.40GHz CPU.

When it is analyzed with our new method, Chebyshev polynomials of the first kind $T_{i}(2 x-1)$ with $i=0,1,2, \cdots, n$ is chosen as the basis functions. An uniform grid $x_{k}=$ $(2 k-1) / 2 N_{x}, k=0,1,2, \cdots, N_{x}, N_{x}+1$ is used to discretize the domain $[0,1]$ where two ghost points $x_{0}$ and $x_{N_{x}+1}$ are also included for implementation of boundary conditions. The operator $\partial^{2} / \partial x^{2}$ is approximated by second-order central difference scheme on the grid, arriving at $\psi\left(x_{k}\right)=\left[\widetilde{\mathcal{L}}\left(T_{i}\right)\right]\left(x_{k}\right)$ on the inner points $\left(k=1,2, \cdots, N_{x}\right)$. Dirichlet boundary condition is approximated with ghost-point condition $\psi\left(x_{0}\right)=-\psi\left(x_{1}\right)$, $\psi\left(x_{N_{x}+1}\right)=-\psi\left(x_{N_{x}}\right)$. After obtaining the interpolant $\psi(x)$, we could use the inner product

$$
\left\langle\psi, T_{j}\right\rangle=\frac{4}{\pi\left(1+\delta_{0 j}\right)} \int_{0}^{1} \frac{\psi(x) T_{j}(2 x-1)}{\sqrt{1-(2 x-1)^{2}}} d x
$$

to obtain $L_{j i}=L_{j i}^{*}$ since $S_{i j}=\delta_{i j}$. In the implementation of the new method on the present problem, the interpolant $\psi(x)$ is evaluated on Chebyshev-Gauss-Lobatto points $\hat{x}_{k}=\left(\cos \left(k \pi / 6 N_{x}\right)+1\right) / 2, k=0,1,2, \cdots, 6 N_{x}$, and the above inner product with the leading $n+1$ Chebyshev polynomials can be evaluated by fast Chebyshev transform (FCT).

In this simple test case, $n=32$ and $N_{x}=200,400,800,1600,3200,6400$ and 12800 are considered. QZ algorithm is used for matrix eigenvalue problem throughout this article. After defining

$$
\Delta \sigma^{(k)}=\sigma^{(k)}-\sigma_{0}^{(k)}
$$

to quantify the errors, the results of the first 10 modes are shown in Fig. 2, together with CPU time spent during matrix formation and eigenvalue computation process. Results of traditional finite difference approach using the same central difference scheme are also shown for comparison in Fig. 2. It can be seen from Fig. 2(a) that for the new method 
and $N_{x}=200$, the relative errors of the first 10 eigenvalues are reasonably low, as low as $0.4 \%$. With the increase of grid density $N_{x}$ in LNS code, the relative errors could be further reduced, and the largest relative error is around $2 \times 10^{-6}$ if $N_{x}=12800$. For finite difference method, the relative errors are smaller but still in the same order as the present method. A perfect scaling of $\left|\Delta \sigma^{(k)} / \sigma_{0}^{(k)}\right| \sim k^{2} N_{x}^{-2}$ could be found for both the new method and finite difference method. This is probably because the relative truncation error of second-order central difference scheme is exactly

$$
\frac{1}{4 !} \frac{\partial^{4} \theta_{0}^{(k)}}{\partial x^{4}}\left(\frac{1}{N_{x}}\right)^{4} /\left(\frac{1}{2 !} \frac{\partial^{2} \theta_{0}^{(k)}}{\partial x^{2}}\left(\frac{1}{N_{x}}\right)^{2}\right) \sim k^{2} N_{x}^{-2}
$$

for the $k$-th eigenmode $\theta_{0}^{(k)}=\sin (k \pi x)$. From Fig. 2(a) and the above scaling analysis, it could be inferred that with a fixed expansion of adequate order, accuracy of the new method can be improved by simply using a denser grid, leaving the matrix size unchanged. For the new method, the matrix size remains to be $(n+1) \times(n+1)=33 \times 33$ regardless of the value of $N_{x}$, while the matrix size will be $N_{x} \times N_{x}$ for the traditional finite difference method. The larger matrix size will increase the CPU time cost in the eigenvalue solver, as shown in Fig. 2(b), where the CPU time spent in matrix formation and eigenvalue computation for both methods are shown. It is obvious that the time cost in the matrix formation increases with $N_{x}$ while it is almost constant for the eigenvalue computation process for the new method. This is reasonable since the LNS solver will cost more time as $N_{x}$ increases while the matrix size of the present method keeps the same as $33 \times 33$. For the traditional finite difference method, the time spent during the matrix forming and the eigenvalue computation will both increase heavily with $N_{x}$. It should be noted that the time cost of the matrix forming process using the finite difference method is less than the new method if $N_{x} \leq 3200$, and we attribute it to the extra step (iv) and the FCT used during the implementation. Nevertheless, the FCT could reduce the time cost when $N_{x}$ is further increased. Since the new method will save a lot of time in the eigenvalue computation, such advantage may allow the new method to outperform the finite difference method in some circumstances. In the present case, the finite difference method requires $0.78 \mathrm{~s}$ to establish and solve the eigenvalue problem for $N_{x}=3200$, while the new method only requires 0.48 s for $N_{x}=12800$ while it can achieve a higher accuracy according to Fig. 2(a).

\subsection{D convection in laterally heated cavity}

Now let's consider a 2D convection in laterally heated cavity, where the upper and lower walls are adiabatic, and the sidewalls with fixed but different temperatures. This is a test case used in Ref. [39]. With the Boussinesq approximation and non-dimensionalization, 
the incompressible NS equation and boundary conditions can be written as

$$
\begin{aligned}
& \nabla \cdot \boldsymbol{u}=0, \\
& \frac{\partial \boldsymbol{u}}{\partial t}+\boldsymbol{u} \cdot \nabla \boldsymbol{u}=-\nabla p+\frac{1}{\sqrt{R a / P r}} \Delta \boldsymbol{u}+\theta \boldsymbol{j}, \\
& \frac{\partial \theta}{\partial t}+\boldsymbol{u} \cdot \nabla \theta=\frac{1}{\sqrt{R a \cdot P r}} \Delta \theta, \\
& x=0: \quad \boldsymbol{u}=0, \quad \theta=0.5, \\
& x=1: \quad \boldsymbol{u}=0, \quad \theta=-0.5, \\
& y=0, \quad A: \boldsymbol{u}=0, \quad \frac{\partial \theta}{\partial y}=0 .
\end{aligned}
$$

During the implementation of the new method, the direct products of first-kind Chebyshev polynomials with maximum order $n_{x}$ and $n_{y}$ respectively are chosen as basis functions:

$$
\begin{aligned}
& \hat{\phi}_{i, j}=T_{i}(2 x-1) T_{j}\left(\frac{2 y-1}{A}\right), \\
& i=0,1,2, \cdots, n_{x} ; \quad j=0,1,2, \cdots, n_{y}
\end{aligned}
$$

for velocity and temperature. Uniform staggered grid with $N_{x} \times N_{y}$ inner temperature grids are used for spatial discretization of $[0,1] \times[0, A]$ with ghost-point treatment for boundary conditions. Second-order central difference scheme are used to approximate the spatial differentials and 2-D discrete cosine transform (DCT) are used to solve the Poisson equation for pressure [40]. Inner product computation is achieved with the same procedure as introduced in Section 3.1.

The cavity height is chosen to be $A=8$, Rayleigh number is $R a=306192$ and Prantl number is $\operatorname{Pr}=0.71$ as used in Ref. [39]. Number of basis functions is $3 \times\left(n_{x}+1\right) \times$ $\left(n_{y}+1\right)=3 \times 31 \times 91$ and grid number for finite difference scheme is $N_{x} \times N_{y}=512 \times 4096$. Steady solution is achieved using the nonlinear NS code with the same algorithm as the LNS code but with some frequency filtering technique [41]. After solving the eigenvalue problem of the matrix with rank $3 \times\left(n_{x}+1\right) \times\left(n_{y}+1\right)$, we obtained the first valid eigenvalue $\sigma_{1}=-6.3721 \times 10^{-5}+1.7085 i$, with a relative deviation of $\left|\Delta \sigma_{1} / \sigma_{1}^{(0)}\right|=3.4 \times 10^{-4}$ from $\sigma_{1}^{(0)}=0+1.70908 i$ obtained using the Galerkin method with $30 \times 90$ basis functions in Ref. [39]. Steady states and eigenmodes are compared with those from Ref. [39] in Fig. 3. Here $\theta_{0}$ and $\psi_{0}$ are steady distribution of temperature and streamfunction respectively. $\left|\widetilde{\theta}_{1}\right|$ and $\left|\widetilde{\psi}_{1}\right|$ are the modulus of complex temperature and streamfunction perturbations of the first eigenmode. 17 contour values are homogeneously arranged between minimum and maximum values of the field as those from Ref. [39], regardless of the amplitudes of computed eigenmodes. It is apparent that the steady solution and the most unstable eigenmode of the temperature and streamfunctions obtained using the present method match very well with the results from Ref. [39], and discrepancies are hardly discernable, demonstrating the accuracy of the present method. 
(a)

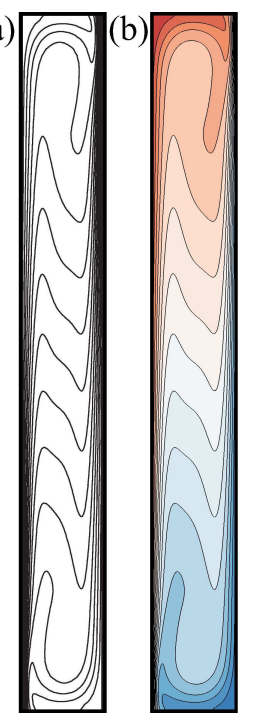

(c)

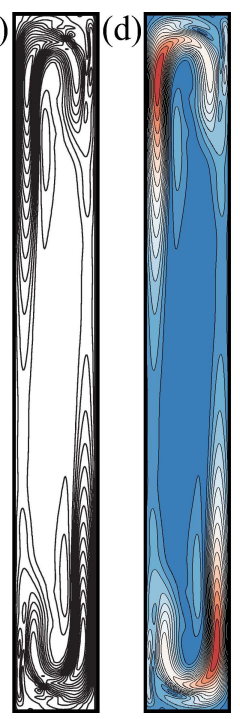

(e)

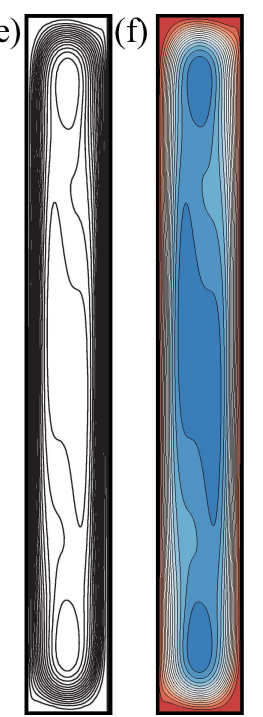

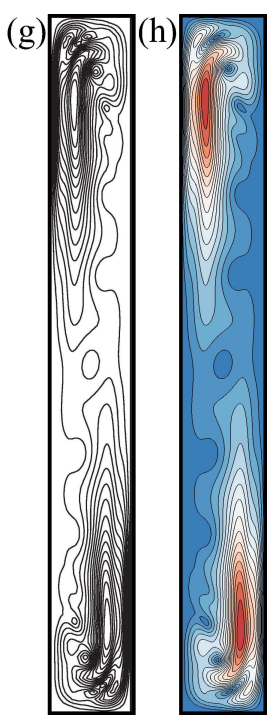

Figure 3: (a,b) Steady temperature $\theta_{0} .(c, d)$ Temperature eigenmode modulus $\left|\widetilde{\theta}_{1}\right| .(e, f)$ Steady streamfunction $\psi_{0}$. (g,h) Streamfunction eigenmode modulus $\left|\widetilde{\theta}_{1}\right|$. (a,c,e,g) are the results in Ref. [39]; (b,d,f,h) are the present results.

\subsection{D Rayleigh-Bénard instability}

The third test case is the 3D Rayleigh-Bénard instability. In this problem, the convection is thermally driven between two infinite planes and has periodic boundary condition in horizontal directions, as shown in Fig. 4. The corresponding 3D thermal convection equation can be written as

$$
\begin{aligned}
& \nabla \cdot \boldsymbol{u}=0, \\
& \frac{\partial \boldsymbol{u}}{\partial t}+\boldsymbol{u} \cdot \nabla \boldsymbol{u}=-\nabla p+\frac{1}{\sqrt{R a / P r}} \Delta \boldsymbol{u}+\theta \boldsymbol{k}, \\
& \frac{\partial \theta}{\partial t}+\boldsymbol{u} \cdot \nabla \theta=\frac{1}{\sqrt{R a \cdot P r}} \Delta \theta, \\
& \boldsymbol{u}(0, y, z)=\boldsymbol{u}\left(L_{x}, y, z\right), \quad \theta(0, y, z)=\theta\left(L_{x}, y, z\right), \\
& \boldsymbol{u}(x, 0, z)=\boldsymbol{u}\left(x, L_{y}, z\right), \quad \theta(x, 0, z)=\theta\left(x, L_{y}, z\right), \\
& z=0: \boldsymbol{u}=0, \quad \theta=0.5, \\
& z=1: \boldsymbol{u}=0, \quad \theta=-0.5 .
\end{aligned}
$$

In the implementation of the present method, the direct products of trigonometric functions ( $x$ and $y$ direction) and first-kind Chebyshev polynomials ( $z$ direction) with maximum order $n_{x}, n_{y}$ and $n_{z}$ respectively are chosen as basis functions for all variables. The finite difference scheme and ghost-point boundary conditions (at lower and upper walls) are the same as Section 3.2. Considering the periodic boundary condition in horizontal 


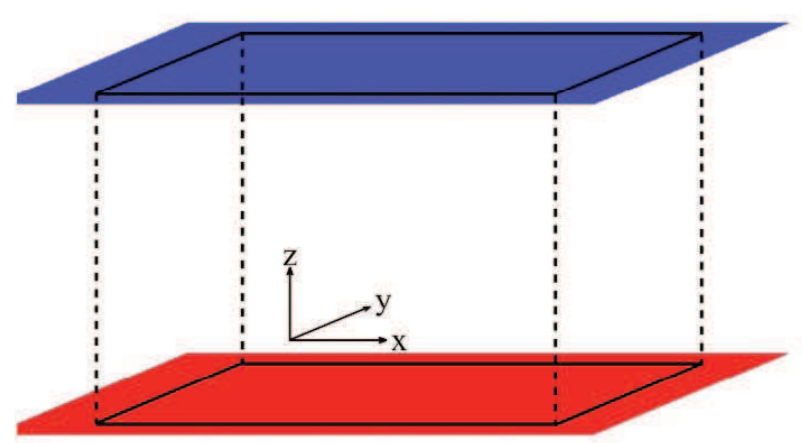

Figure 4: Sketch of the 3-D Rayleigh-Bénard convection with horizontally periodic boundary conditions.

directions, the Poisson solver is based on DCT in $z$ direction and fast Fourier transform (FFT) in $x$ and $y$ directions. The inner product could be realized by doing FFT ( $x$ and $y$ direction) and DCT ( $z$ direction), which is similar as Section 3.1.

The parameters are chosen as $\operatorname{Ra}=2 \times 10^{4}, \operatorname{Pr}=1, L_{x}=L_{y}=1$. The steady state is chosen to be static, which is $\boldsymbol{u}_{0}=0, \theta_{0}=0.5-z$. The number of basis functions is $4 \times\left(2 n_{x}+\right.$ $1) \times\left(2 n_{y}+1\right) \times\left(n_{z}+1\right)=4 \times 5 \times 5 \times 21$ and the grid number of finite difference scheme is $N_{x} \times N_{y} \times N_{z}=128 \times 128 \times 128$. We define horizontal average being $\langle\cdot\rangle_{H}$ and vertical average being $\langle\cdot\rangle_{z}$. The first 8 eigenmodes are obtained, arranged into 2 groups, and decomposed into horizontal modes

$$
f_{i, j}(x, y)=\frac{\left\langle\widetilde{\theta}_{i, j}(x, y, z)\right\rangle_{z}}{\max \left[\left\langle\widetilde{\theta}_{i, j}(x, y, z)\right\rangle_{z}\right]}
$$

and vertical amplitudes

$$
\begin{aligned}
& \Theta_{i, j}(z) \triangleq\left\langle\widetilde{\theta}_{i, j}(x, y, z) / f_{i, j}(x, y)\right\rangle_{H}, \\
& W_{i, j}(z) \triangleq\left\langle\widetilde{w}_{i, j}(x, y, z) / f_{i, j}(x, y)\right\rangle_{H} .
\end{aligned}
$$

Fig. 5 shows the horizontal modes of the first 8 eigenmodes. The horizontal modes of the first 4 eigenmodes seem to be linear combinations of $\sin (2 \pi x+\beta)$ and $\sin (2 \pi y+\gamma)$ while the next 4 horizontal modes seem to be linear combinations of $\sin (2 \pi x+\beta) \sin (2 \pi y+\gamma)$. Since there are 4 eigenmodes corresponding to one eigenvalue, any linear combination of the 4 eigenmodes could be an eigenmode corresponding to the same eigenvalue. This indicates that the computed eigenmodes are not necessary to be orthogonal.

According to linear stability theory, the eigenmodes should satisfy

$$
\begin{aligned}
& \tilde{w}=f(x, y) W(z), \\
& \tilde{\theta}=f(x, y) \Theta(z), \\
& \Delta_{H} f+a^{2} f=0, \quad a=\sqrt{(2 m \pi)^{2}+(2 n \pi)^{2}},
\end{aligned}
$$



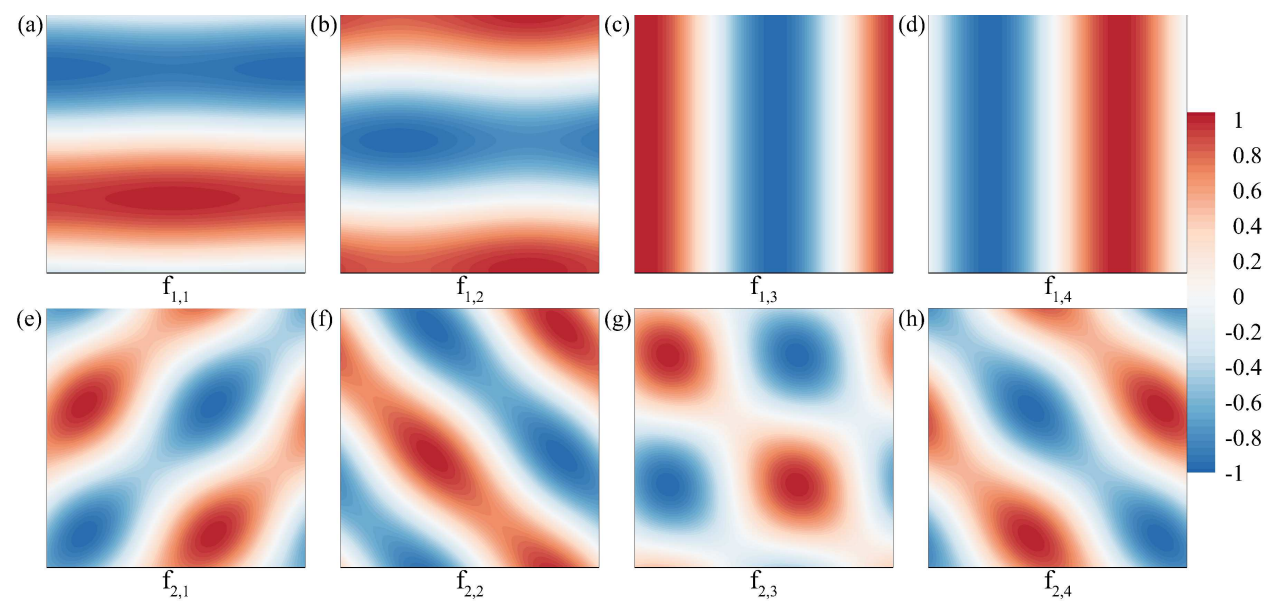

Figure 5: Horizontal modes of the first 8 eigenmodes.

where $\Delta_{H}=\partial^{2} / \partial x^{2}+\partial^{2} / \partial y^{2}$ and $m, n=1,2,3, \cdots$. It is seen that $a=2 \pi$ for the upper 4 eigenmodes while $a=2 \sqrt{2} \pi$ for the lower 4 eigenmodes shown in Fig. 5 respectively.

Define the errors based on $L^{1}$ and $L^{2}$ norm

$$
\begin{aligned}
& \operatorname{err}_{1}\left[q^{(0)}, q\right]=\left(q-q^{(0)}\right) / \max \left(q^{(0)}\right), \\
& \overline{\operatorname{err}}_{2}\left[q^{(0)}, q\right]=\sqrt{\int_{V}\left|q-q^{(0)}\right|^{2} d V / \int_{V}\left|q^{(0)}\right|^{2} d V},
\end{aligned}
$$

and it is shown that the deviations from variable separation and the horizontal Laplacian eigenvalue results are small:

$$
\begin{aligned}
& \overline{\operatorname{err}}_{2}\left[f_{i, j}(x, y) W_{i, j}(z), \tilde{w}_{i, j}\right]<2 \times 10^{-13}, \\
& \overline{\operatorname{err}}_{2}\left[f_{i, j}(x, y) \Theta_{i, j}(z), \tilde{\theta}_{i, j}\right]<3 \times 10^{-13}, \\
& \overline{\operatorname{err}}_{2}\left[-a_{i}^{2} f_{i, j}(x, y), \Delta_{H} f_{i, j}(x, y)\right]<5 \times 10^{-11} .
\end{aligned}
$$

To further examine the accuracy of the present result, Chebyshev collocation method on 65 Chebyshev-Gauss-Lobatto points is applied to solve the 6-th order Orr-Sommerfeld equation, obtaining the reference vertical amplitudes. The relative deviation of eigenvalues $\left|\Delta \sigma_{i, j} / \sigma_{i}{ }^{(0)}\right|<1 \times 10^{-4}$. In Fig. 6 the deviations between vertical amplitudes $\Theta(z)$ and $W(z)$ are shown. The results showed satisfactory accuracy of the present method in $3 \mathrm{D}$ case. In addition, Fig. 6 shows that the computed eigenmodes corresponding to the same eigenvalue are very close to each other in the vertical amplitudes. Such consistency is probably due to the homogeneity of both the finite difference method and spectral expansion in the $x$ and $y$ directions. 

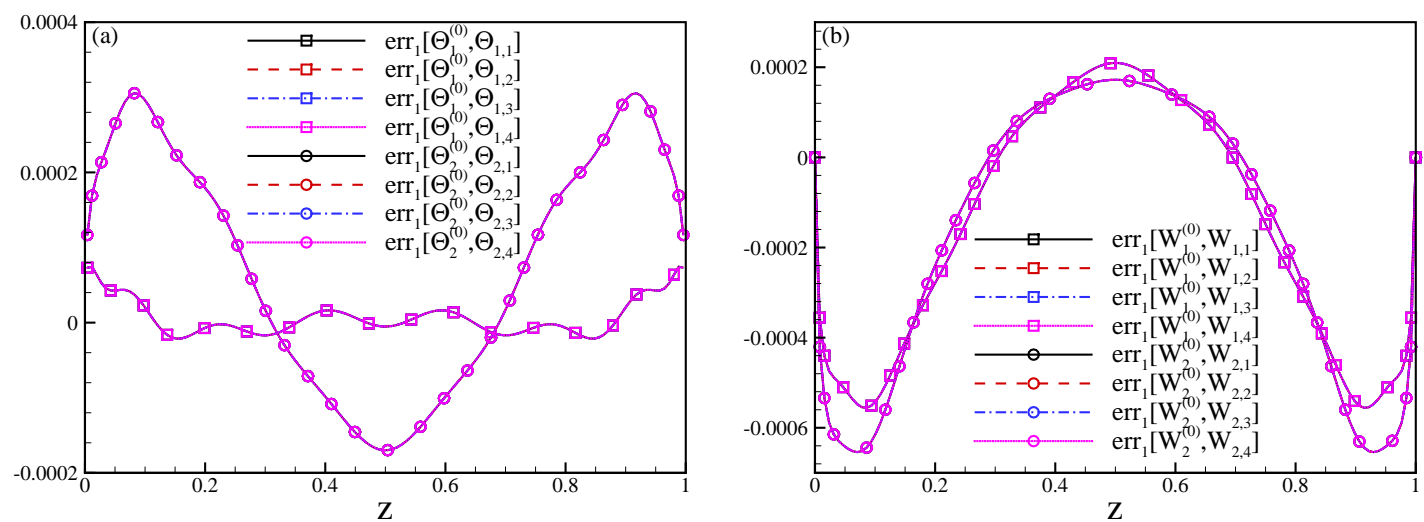

Figure 6: Deviation of vertical amplitudes between the present method and Chebyshev collocation method. (a) Temperature perturbation. (b) Vertical velocity perturbation. The superscript "0" denotes the results from Chebyshev collocation method.

\subsection{D mixed convection flow}

Previous cases have shown the reliability of the new method, and it is necessary to demonstrate the effectiveness of the new method in a problem with relatively complex boundary conditions. Let's consider the mixed convection flow introduced in Ref. [42]. As shown in Fig. 7, the upper wall is adiabatic and the lower wall has both adiabatic and isothermal regions. Such problem with mixed and non-homogeneous boundary condition simulates the winds or currents flowing past solid ground with non-uniform temperature, thermal capacity and thermal conductivity.

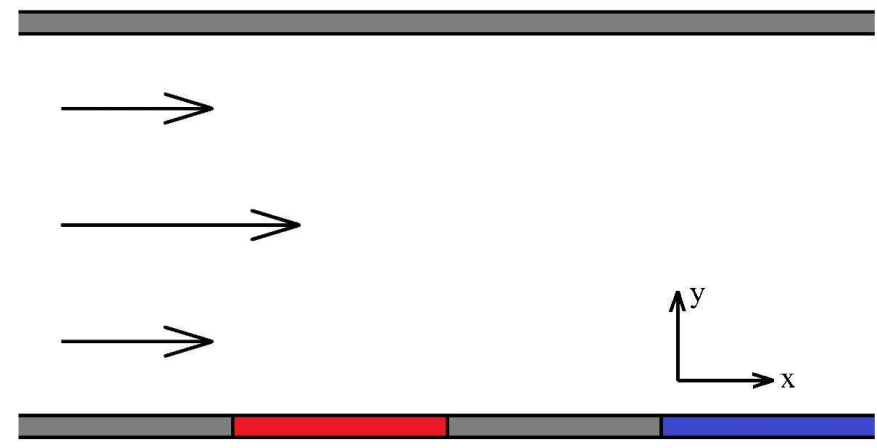

Figure 7: Sketch of 2-D mixed convection flow.

The non-dimensionalized governing equations and boundary conditions can be written as

$$
\nabla \cdot \boldsymbol{u}=0
$$




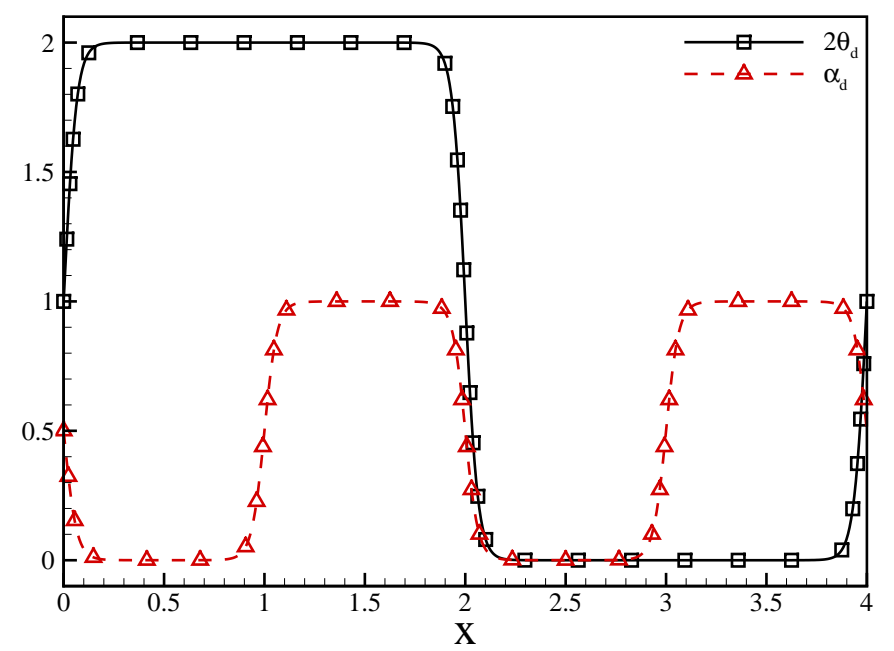

Figure 8: Temperature and conductivity parameter on the lower wall.

$$
\begin{aligned}
& \frac{\partial \boldsymbol{u}}{\partial t}+\boldsymbol{u} \cdot \nabla \boldsymbol{u}=-\nabla p+\frac{1}{R e} \Delta \boldsymbol{u}+\theta j \\
& \frac{\partial \theta}{\partial t}+\boldsymbol{u} \cdot \nabla \theta=\frac{1}{\operatorname{RePr}} \Delta \theta \\
& y=0: \quad \boldsymbol{u}=0, \quad \alpha_{d}\left(\theta-\theta_{d}\right)+\left(1-\alpha_{d}\right) \frac{\partial \theta}{\partial n}=0, \\
& y=2: \quad \boldsymbol{u}=0, \quad \frac{\partial \theta}{\partial n}=0, \\
& \boldsymbol{u}(0, y)=\boldsymbol{u}\left(L_{x}, y\right), \quad \theta(0, y)=\theta\left(L_{x}, y\right) \\
& \theta_{d}=\left[\tanh \left(10 \sin \frac{\pi x}{2}\right)+1\right] / 2 \\
& \alpha_{d}=[-\tanh (5 \sin (\pi x))+1] / 2
\end{aligned}
$$

with $\alpha_{d}$ and $\theta_{d}$ being continuous, which is suitable for the spectral expansion in the $x$ direction. $\theta_{d}(x)$ and $\alpha_{d}(x)$ are shown in Fig. 8.

It is troublesome to use classic Galerkin method for such problem because the basis functions for temperature cannot be given analytically. However, with the present method, all variables can be expanded with basis functions being direct products of trigonometric functions and first-kind Chebyshev polynomials. The treatment of complex boundary conditions are left to the LNS code. The LNS code and interpolation procedure in the vertical and horizontal direction are similar as those in Section 3.3.

In the implementation, the parameters are chosen as $\operatorname{Re}=60, \operatorname{Ra}=1 \times 10^{5}, \operatorname{Pr}=1, L_{x}=4$, $n_{x} \times n_{y}=81 \times 30, N_{x} \times N_{y}=512 \times 256$. Steady solution is computed with the same procedure as Section 3.2. Reference results of the first eigenvalue and eigenmode are obtained with the time-advancing algorithm, and the first eigenvalue is $0.268900+3.59830 i$. The first 

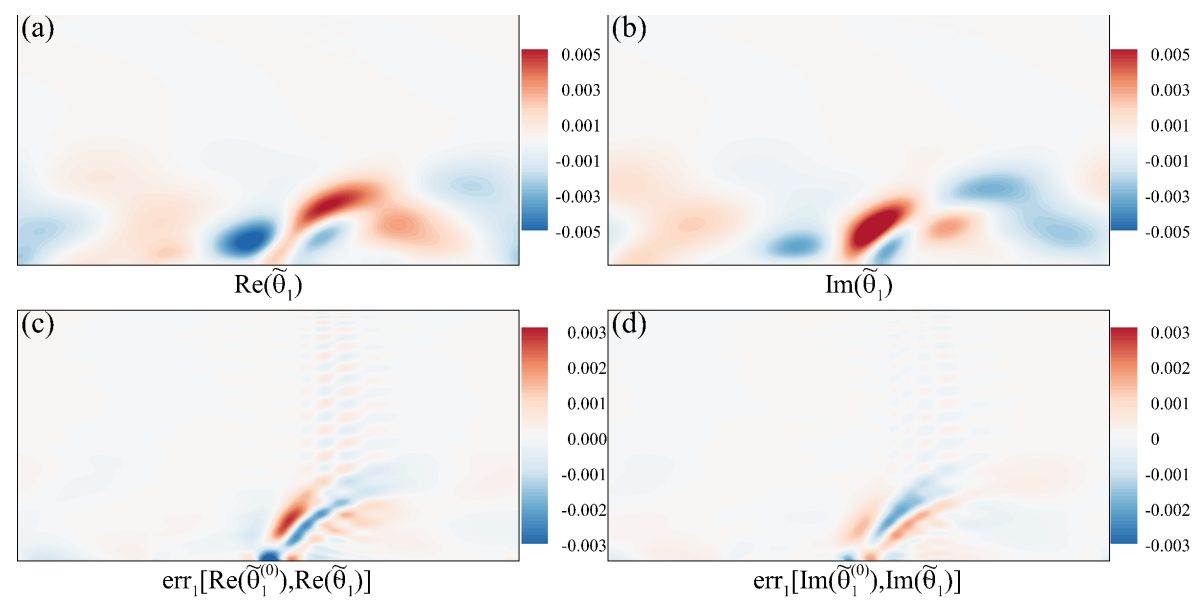

Figure 9: $(a, b)$ : Real and imaginary part of the first temperature eigenmode $\widetilde{\theta}_{1}$ from the present new method; (c): Relative deviations between time-stepping results $\operatorname{Re}\left(\widetilde{\theta}_{1}^{(0)}\right)$ and the result $\operatorname{Re}\left(\widetilde{\theta}_{1}\right)$ from the new method.

(d) Relative deviations between $\operatorname{Im}\left(\widetilde{\theta}_{1}^{(0)}\right)$ and $\operatorname{Im}\left(\widetilde{\theta}_{1}\right)$.

eigenvalue from the eigenvalue problem of the matrix constructed with the new method is $0.268835+3.59827 i$, and the relative deviation is less than $2.0 \times 10^{-5}$. Fig. $9(a, b)$ show the real and imaginary part of the temperature eigenmode obtained from the new method and Fig. 9(c,d) show their relative deviations from the time-advancing results. Relative deviation of order $10^{-3}$ is obtained, which shows a surprising accuracy of the new matrixforming method even for non-traditional boundary conditions. Deviations are relatively larger when the flow on the hot wall comes to the adiabatic wall. This is probably because the hot boundary layer separates at the end of the hot wall, and the sharp transition of boundary condition makes the local field more difficult to resolve.

\subsection{Lid-driven flow in L-shaped cavity}

Finally, in order to show the reliability of the new method for complex geometry, let's consider the lid-driven flow in L-shaped cavity $[43,44]$. Inside an infinitely long channel with L-shaped cross section $\mathcal{D}=\overline{\mathcal{D}} \times(-\infty, \infty)=(([0,1] \times[0,1]) \backslash((0,0.5) \times(0,0.5))) \times(-\infty, \infty)$, the fluid is driven along $x$ direction by the top wall $y=1$. The basic equation and boundary conditions can be written as

$$
\begin{aligned}
& \nabla \cdot \boldsymbol{u}=0, \\
& \frac{\partial \boldsymbol{u}}{\partial t}+\boldsymbol{u} \cdot \nabla \boldsymbol{u}=-\nabla p+\frac{1}{\operatorname{Re}} \Delta \boldsymbol{u}, \\
& \partial \mathcal{D} \backslash([0,1] \times\{1\} \times(-\infty, \infty)): \boldsymbol{u}=0, \\
& {[0,1] \times\{1\} \times(-\infty, \infty): \boldsymbol{u}=\left[1-(2 x-1)^{18}\right]^{2} \boldsymbol{e}_{x} .}
\end{aligned}
$$


With a $2 \mathrm{D}$ base flow $\boldsymbol{u}(x, y)=\left(u_{0}, v_{0}, 0\right)$, the flow could be unstable and the evolution of the infinitesimal perturbation with given wavenumber $\beta$ in $z$ direction satisfies a $2 \mathrm{D}$ equation which could be solved with an adapted 2D LNS code.

Unlike the multi-domain strategy used in Ref. [44], we attempt to expand the 2D fields in the L-shaped cross section with simple combination of Chebyshev polynomials

$$
\begin{aligned}
& \hat{\phi}_{i, j}=T_{i}(\xi) T_{j}(\eta), \\
& i=0,1,2, \cdots, n_{\xi} ; \quad j=0,1,2, \cdots, n_{\eta}
\end{aligned}
$$

after the variable transformation. First, mapping $(x, y)$ to the polar coordinates:

$$
\begin{aligned}
& r(x, y)=\left(x^{2}+y^{2}\right)^{1 / 2}, \\
& \theta(x, y)=\tan ^{-1}(y / x),
\end{aligned}
$$

and specifying the maximum and minimum $r$ reached in $\overline{\mathcal{D}}$ :

$$
\begin{aligned}
& r_{o}(\theta)=\sec (\pi / 4-|\pi / 4-\theta|), \\
& r_{i}(\theta)=\sec (\pi / 4-|\pi / 4-\theta|) / 2 .
\end{aligned}
$$

Then the variables $(\xi, \eta)$ could be obtained from $(r, \theta)$ :

$$
\begin{aligned}
& \xi(r, \theta)=2\left[r-\left(r_{o}+r_{i}\right) / 2\right] /\left(r_{o}-r_{i}\right), \\
& \eta(r, \theta)=f_{s}^{-1}(4 \theta / \pi-1),
\end{aligned}
$$

where $f_{s}$ is a monotone mapping from $[-1,1]$ to $[-1,1]$. In order to resolve the flow around the corner at $(x, y)=(0.5,0.5)$ and $(x, y)=(1,1), f_{s}$ should contain proper stretching there and it is defined as

$$
f_{s}(\zeta)=\frac{\tanh \left(a_{2}\left(\zeta-a_{1}\right)\right)+\tanh \left(a_{2}\left(\zeta+a_{1}\right)\right)}{\tanh \left(a_{2}\left(1-a_{1}\right)\right)+\tanh \left(a_{2}\left(1+a_{1}\right)\right)}
$$

with $a_{1}=0.8, a_{2}=3$.

The order of basis functions is chosen as $\left(n_{\xi}+1\right) \times\left(n_{\eta}+1\right)=21 \times 49$. The resolution of the Chebyshev polynomials is indicated by Fig. 10. Fig. 10(a) shows conceptual mesh $\left(x\left(\xi_{i}, \eta_{j}\right), y\left(\xi_{i}, \eta_{j}\right)\right)$ defined by the Chebyshev-Gauss-Lobatto points $\xi_{i}$ and $\eta_{j}$, and Fig. 10(b) shows the last (highest-order) basis function $\hat{\phi}_{n_{\tilde{\xi}}, n_{\eta}}(\xi(x, y), \eta(x, y))$.

The Reynolds number is chosen as $R e=650$ and the wavenumber is chosen as $\beta=9.7$. The LNS code is based on the second-order central difference scheme on homogeneous grid with $N_{x} \times N_{y}=256 \times 256$. The inner product in the $2 \mathrm{D}$ domain is defined as

$$
\left\langle\psi_{1}, \psi_{2}\right\rangle=\int_{\bar{D}} \psi_{1} \cdot \psi_{2} d s
$$

and the integral is computed numerically on the LNS grid. 
(a)

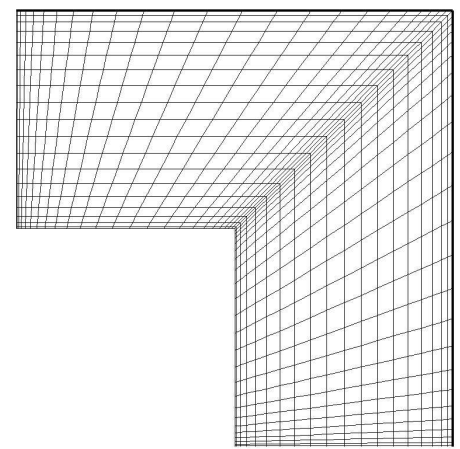

(b)

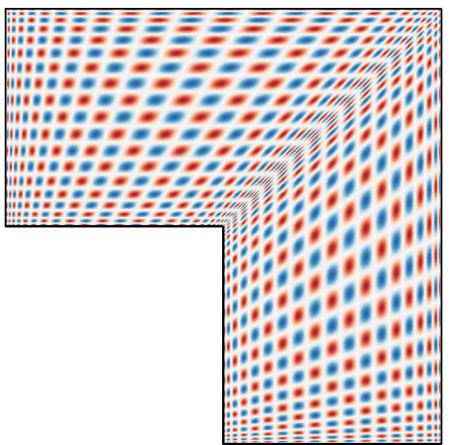

Figure 10: Sketch of the spectral expansion in L-shaped cavity. (a) Conceptual mesh defined by ChebyshevGauss-Lobatto points. (b) Contour of the highest-order basis function used.
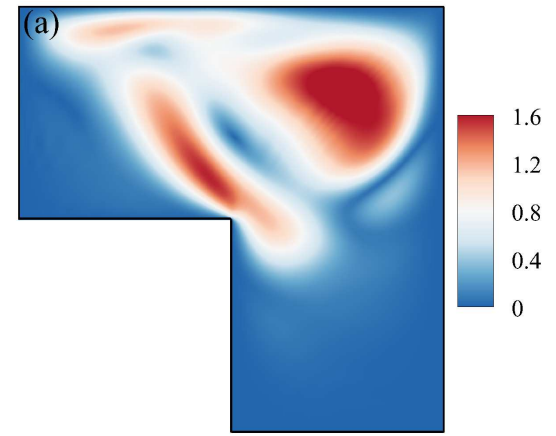

$\left|\widetilde{\mathrm{u}}_{1}\right|$

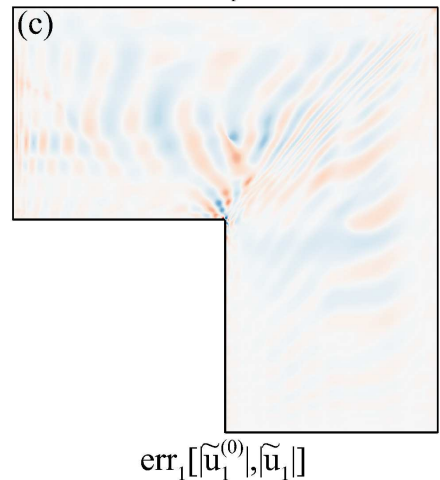

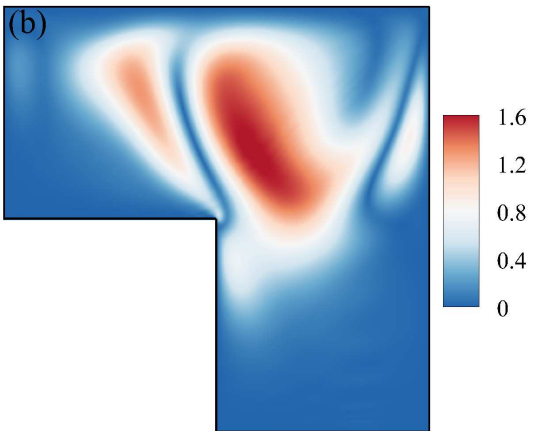

$\widetilde{\mathrm{v}_{1} \mid}$

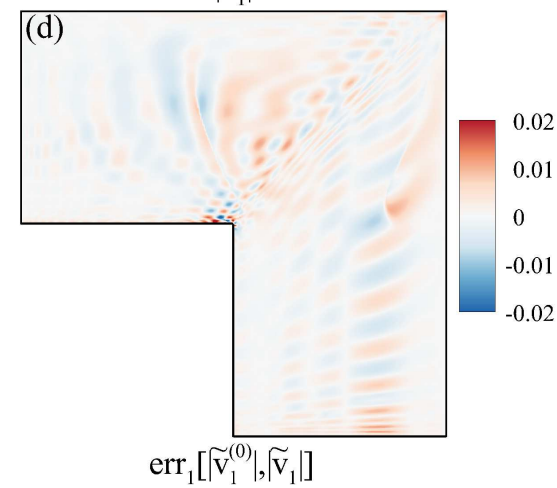

Figure 11: (a) Amplitude of the first $u$ eigenmode $\widetilde{u}_{1}$ from the present new method; (b) Amplitude of the first $v$ eigenmode $\widetilde{v}_{1}$ from the present new method; (c) Relative deviations between time-stepping results $\left|\widetilde{u}_{1}^{(0)}\right|$ and the result $\left|\widetilde{u}_{1}\right|$ from the new method. (d) Relative deviations between $\left|\widetilde{v}^{(0)}\right|$ and $\left|\widetilde{v}_{1}\right|$.

The reference result of the leading eigenmode is obtained with the time-advancing method. The first eigenvalue obtained with the new method is $0.0037+1.0654 i$ and the reference value from the time-advancing method is $0.0041+1.0649 i$, with a relative de- 
viation of $6.0 \times 10^{-4}$. Both values only have a relative deviation of 0.02 from the result in Ref. [44]. The amplitudes of the computed first eigenmode are shown in Fig. 11(a,b). Fig. 11(c,d) show the corresponding relative deviations of order $10^{-2}$ from the reference result. Clearly, the present method could obtain a very good estimation on both the eigenvalues and the eigenmodes in this L-shaped cavity.

\section{Conclusion}

In order to analyze the global linear instability problem with little mathematics and more flexibility, the present work introduces a new CFD-aided matrix-forming method which combines the merits from both the high-order and low-order methods. The new method adopts the global Galerkin framework while the LNS code is used to obtain the transferring matrix. No requirement of incompressibility or boundary condition is needed for basis functions, allowing a wider choice of boundary conditions and other physical constraints within the frame of the Galerkin method. The new method is validated in 1D, 2D and 3D test cases with regular or complex boundary conditions. The eigenvalues along with eigenmodes obtained with the new method are compared with those reference results, and remarkable consistency can be obtained for both the eigenvalues and eigenmodes.

For a wide range of geometries available for spectral expansion, such as rectangle, ellipsoid, cylinder and analytical airfoils, global linear instability can be analyzed using the present CFD-aided Galerkin method. With geometry and spectral expansion fixed, no extra mathematical derivation is required for complex boundary conditions, given an LNS code capable of resolving them.

\section{Acknowledgments}

The work is supported by the National Science Foundation of China (NSFC Grants No. 11822208, No. 11772297, No. 91752201, and No. 91752202). Z.H. Xia would also like to thank the support from the Fundamental Research Funds for the central Universities.

\section{References}

[1] P. Drazin, W. Reid, Hydrodynamic stability, Cambridge Univ, Press, Cambridge (1981) 8-14.

[2] X. Wu, Z. Heng, Linear instability of turbulent boundary layer as a mechanism for the generation of large scale coherent structures, Chinese Science Bulletin (20) (1989) 1685-1688.

[3] Y. Bai, Y. Yang, The dynamic stability of the flow in a meander channel, Science China Technological Sciences 54 (4) (2011) 931-940.

[4] X. Sun, J. Peng, K. Zhu, Stability of core-annular flow of power-law fluids in the presence of interfacial surfactant, Science China Physics, Mechanics and Astronomy 53 (5) (2010) 933943. 
[5] T. Herbert, Parabolized stability equations, Annual Review of Fluid Mechanics 29 (1) (1997) 245-283.

[6] G. Xu, Z. Xiao, S. Fu, Analysis of the secondary instability of the incompressible flows over a swept wing, Science China Physics, Mechanics and Astronomy 54 (4) (2011) 724-736.

[7] J. Ren, S. Fu, Floquet analysis of fundamental, subharmonic and detuned secondary instabilities of Görtler vortices, Science China Physics, Mechanics and Astronomy 57 (3) (2014) $555-561$.

[8] R. Pierrehumbert, S. Widnall, The two-and three-dimensional instabilities of a spatially periodic shear layer, Journal of Fluid Mechanics 114 (1982) 59-82.

[9] J. de Vicente, E. Valero, L. González, V. Theofilis, Spectral multi-domain methods for biglobal instability analysis of complex flows over open cavity configurations, in: 36th AIAA Fluid Dynamics Conference and Exhibit, 2877, 2006.

[10] C. Canuto, M. Y. Hussaini, A. Quarteroni, T. A. Zang, Spectral methods, Springer, 2006.

[11] P. Demaret, M. Deville, Chebyshev collocation solutions of the Navier-Stokes equations using multi-domain decomposition and finite element preconditioning, Journal of Computational Physics 95 (2) (1991) 359-386.

[12] C. H. Amon, A. T. Patera, Numerical calculation of stable three-dimensional tertiary states in grooved-channel flow, Physics of Fluids A: Fluid Dynamics 1 (12) (1989) 2005-2009.

[13] W. He, R. d. S. Gioria, J. M. Pérez, V. Theofilis, Linear instability of low Reynolds number massively separated flow around three NACA airfoils, Journal of Fluid Mechanics 811 (2017) 701-741.

[14] V. Kitsios, D. Rodríguez, V. Theofilis, A. Ooi, J. Soria, BiGlobal stability analysis in curvilinear coordinates of massively separated lifting bodies, Journal of Computational Physics 228 (19) (2009) 7181-7196.

[15] R. Pierrehumbert, Universal short-wave instability of two-dimensional eddies in an inviscid fluid, Physical review letters 57 (17) (1986) 2157.

[16] F. Chatelin, Spectral approximation of linear operators, SIAM, 2011.

[17] A. Y. Gelfgat, Different modes of Rayleigh-Bénard instability in two-and three-dimensional rectangular enclosures, Journal of Computational Physics 156 (2) (1999) 300-324.

[18] V. Theofilis, Globally unstable basic flows in open cavities, in: 6th Aeroacoustics Conference and Exhibit, 1965, 2000.

[19] E. Ferrer, J. de Vicente, E. Valero, Low cost 3D global instability analysis and flow sensitivity based on dynamic mode decomposition and high-order numerical tools, International Journal for Numerical Methods in Fluids 76 (3) (2014) 169-184.

[20] F. Meseguer-Garrido, J. De Vicente, E. Valero, V. Theofilis, On linear instability mechanisms in incompressible open cavity flow, Journal of Fluid Mechanics 752 (2014) 219-236.

[21] S. Hoyas, H. Herrero, A. Mancho, Thermocapillar and thermogravitatory waves in a convection problem, Theoretical and Computational Fluid Dynamics 18 (2-4) (2004) 309-321.

[22] J. Chen, H. Chen, G. Zhang, S. Fu, Stability of flow between rotating cylinders with axial buoyancy effect, Science in China Series G: Physics, Mechanics and Astronomy 49 (5) (2006) 564-575.

[23] C. J. Mack, P. J. Schmid, A preconditioned Krylov technique for global hydrodynamic stability analysis of large-scale compressible flows, Journal of Computational Physics 229 (3) (2010) 541-560.

[24] J. Crouch, A. Garbaruk, D. Magidov, Predicting the onset of flow unsteadiness based on global instability, Journal of Computational Physics 224 (2) (2007) 924-940.

[25] F. Gómez, S. L. Clainche, P. Paredes, M. Hermanns, V. Theofilis, Four decades of studying 
global linear instability: Progress and challenges, AIAA journal 50 (12) (2012) 2731-2743.

[26] Q.-Z. Feng, Instability and chaos in 2-dimensional viscous flow past a circular cylinder, Chinese Science Bulletin (7) (1991) 5.

[27] B. Xun, K. Li, W. Hu, Instability of thermocapillary flow in liquid layers under microgravity, Science China Physics, Mechanics and Astronomy 55 (4) (2012) 684-692.

[28] B. Wang, Z. Guo, D. Ma, D. Sun, Instabilities and pattern evolution in a vertically heated annulus, Science China Physics, Mechanics and Astronomy 56 (2) (2013) 257-262.

[29] Y. Ding, M. Kawahara, Linear stability of incompressible flow using a mixed finite element method, Journal of Computational Physics 139 (2) (1998) 243-273.

[30] R. Pawlowski, A. Salinger, J. Shadid, T. Mountziaris, Bifurcation and stability analysis of laminar isothermal counterflowing jets, Journal of Fluid Mechanics 551 (2006) 117-139.

[31] L. M. González, R. Gómez-Blanco, V. Theofilis, Eigenmodes of a counter-rotating vortex dipole, AIAA journal 46 (11) (2008) 2796-2805.

[32] F. Gómez, R. Gómez, V. Theofilis, On three-dimensional global linear instability analysis of flows with standard aerodynamics codes, Aerospace Science and Technology 32 (1) (2014) 223-234.

[33] Q. Liu, F. Gómez, J. Perez, V. Theofilis, Instability and sensitivity analysis of flows using

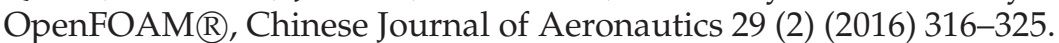

[34] J. M. Pérez, A. Aguilar, V. Theofilis, Lattice Boltzmann methods for global linear instability analysis, Theoretical and Computational Fluid Dynamics 31 (5-6) (2017) 643-664.

[35] L. Vienne, S. Marie, F. Grasso, Simulation of Viscous Fingering Instability by the Lattice Boltzmann Method, in: AIAA Aviation 2019 Forum, 3432, 2019.

[36] P. Paredes, M. Hermanns, S. Le Clainche, V. Theofilis, Order 104 speedup in global linear instability analysis using matrix formation, Computer Methods in Applied Mechanics and Engineering 253 (2013) 287-304.

[37] L. E. Eriksson, A. Rizzi, Computer-aided analysis of the convergence to steady state of discrete approximations to the Euler equations, Journal of Computational Physics 57 (1) (1985) $90-128$.

[38] V. Theofilis, Global linear instability, Annual Review of Fluid Mechanics 43 (2011) 319-352.

[39] A. Y. Gelfgat, Stability and slightly supercritical oscillatory regimes of natural convection in a 8: 1 cavity: solution of the benchmark problem by a global Galerkin method, International Journal for Numerical Methods in Fluids 44 (2) (2004) 135-146.

[40] Y.-Z. Zhang, Y. Bao, Direct solution method of efficient large-scale parallel computation for 3D turbulent Rayleigh-Benard convection, Acta Physica Sinica 64 (15).

[41] E. Åkervik, L. Brandt, D. S. Henningson, J. Hœpffner, O. Marxen, P. Schlatter, Steady solutions of the Navier-Stokes equations by selective frequency damping, Physics of fluids 18 (6) (2006) 068102.

[42] A. Tangborn, A two-dimensional instability in a mixed convection flow with spatially periodic temperature boundary conditions, Physics of Fluids A: Fluid Dynamics 4 (7) (1992) $1583-1586$.

[43] C. Oosterlee, P. Wesseling, A. Segal, E. Brakkee, Benchmark solutions for the incompressible Navier-Stokes equations in general co-ordinates on staggered grids, International Journal for Numerical Methods in Fluids 17 (4) (1993) 301-321.

[44] J. de Vicente Buendía, E. Valero, Spectral Multi-Domain Method for the Global Instability Analysis of Complex Cavity Flows, Ph.D. thesis, School Aeronaut., Univ. Politéc. Madrid, 2010. 